\title{
Erratum to: Bortezomib represses HIF-1 $\alpha$ protein expression and nuclear accumulation by inhibiting both PI3K/Akt/TOR and MAPK pathways in prostate cancer cells
}

\author{
C. D. Befani - P. J. Vlachostergios • E. Hatzidaki • \\ A. Patrikidou $\cdot$ S. Bonanou • G. Simos • \\ C. N. Papandreou • P. Liakos
}

Published online: 17 April 2013

(C) Springer-Verlag Berlin Heidelberg 2013

\section{Erratum to: J Mol Med (Berl). 2012 Jan;90(1):45-54 DOI 10.1007/s00109-011-0805-8}

It has been brought to our attention as authors that two panels in Fig. 1b, showing blots for p-MAPK and MAPK in LNCaP cells, look identical. The corrected version is given below. Another error occurred in Fig. 4b, where the panels for MAPK expression in the nucleus and the cyto- plasm of LNCaP cells were identical. The authors state that these errors occured inadvertently during electronic arrangement of the figures. The MAPK panel in Fig. $1 \mathrm{~b}$ (right side) and the nuclear MAPK panels in Fig. 4b were replaced appropriately and the corrected figures are given below. The authors apologize for this oversight and state that the findings and conclusions of their publication are not affected by these changes.

The online version of the original article can be found at http://dx.doi.org/ 10.1007/s00109-011-0805-8.

C. D. Befani $\cdot$ S. Bonanou $\cdot$ G. Simos $\cdot$ P. Liakos

Laboratory of Biochemistry, Faculty of Medicine,

University of Thessaly, Biopolis, 41110 Larissa, Greece

P. J. Vlachostergios · E. Hatzidaki • A. Patrikidou •

C. N. Papandreou

Department of Medical Oncology, University Hospital of Larissa,

University of Thessaly, Biopolis, 41110 Larissa, Greece

G. Simos $\cdot$ P. Liakos

Institute of Biomedical Research and Technology (BIOMED),

51 Papanastasiou str,

41222 Larissa, Greece

\section{P. Liakos $(\bowtie)$}

Faculty of Medicine, Laboratory of Biochemistry,

University of Thessaly, Biopolis, 41110 Larissa, Greece

e-mail: pliakos@med.uth.gr

\section{N. Papandreou $(\bowtie)$}

Department of Medical Oncology, Faculty of Medicine,

University Hospital of Larissa, University of Thessaly, Biopolis,

41110 Larissa, Greece

e-mail: cpapandreou@med.uth.gr 
a

LNCaP

Hypoxia $\left(1 \% \mathrm{O}_{2}\right)$

\begin{tabular}{lllllll}
0 & 2 & 4 & 8 & 16 & 24 & 48 \\
\hline
\end{tabular} HIF-1a $\square=0$

Actin $-\infty-\infty-\infty$

b

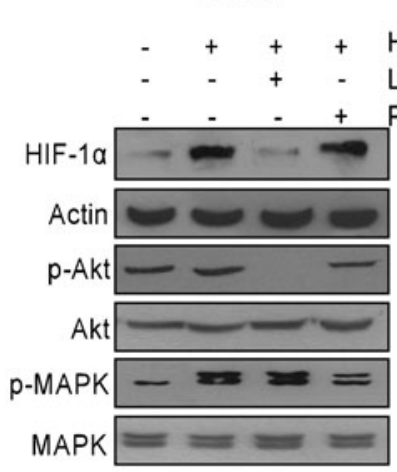

PC3

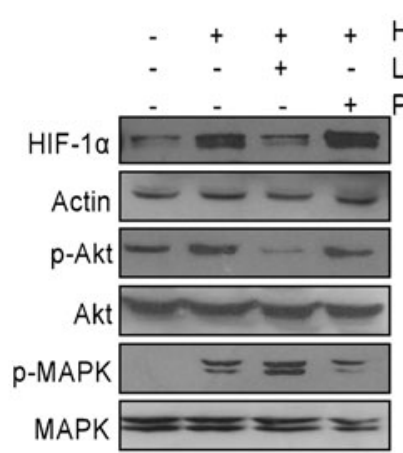

C

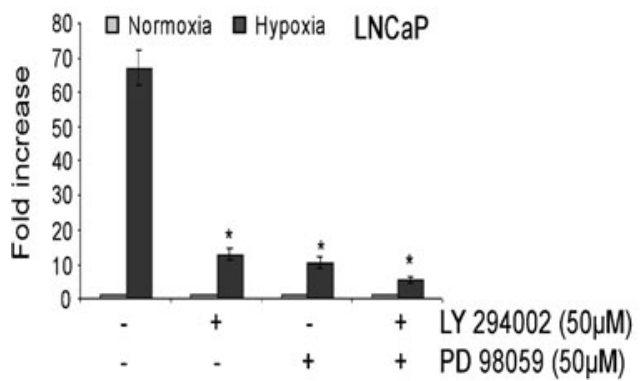

PC3

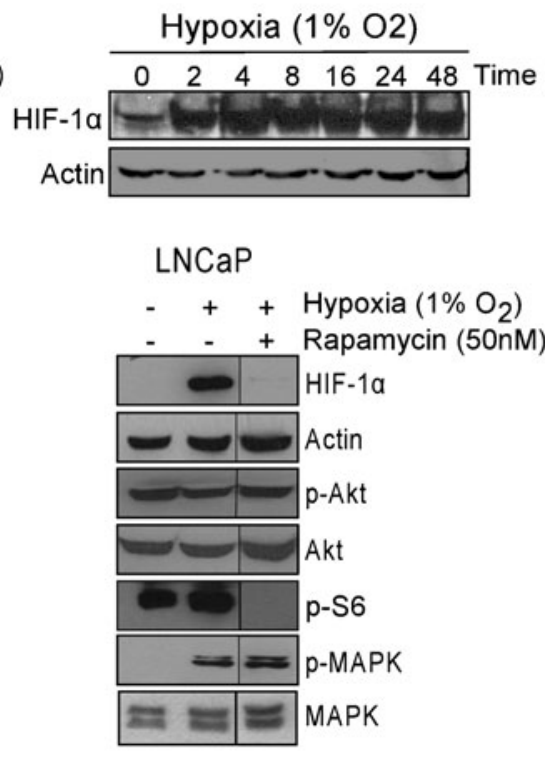

PC3
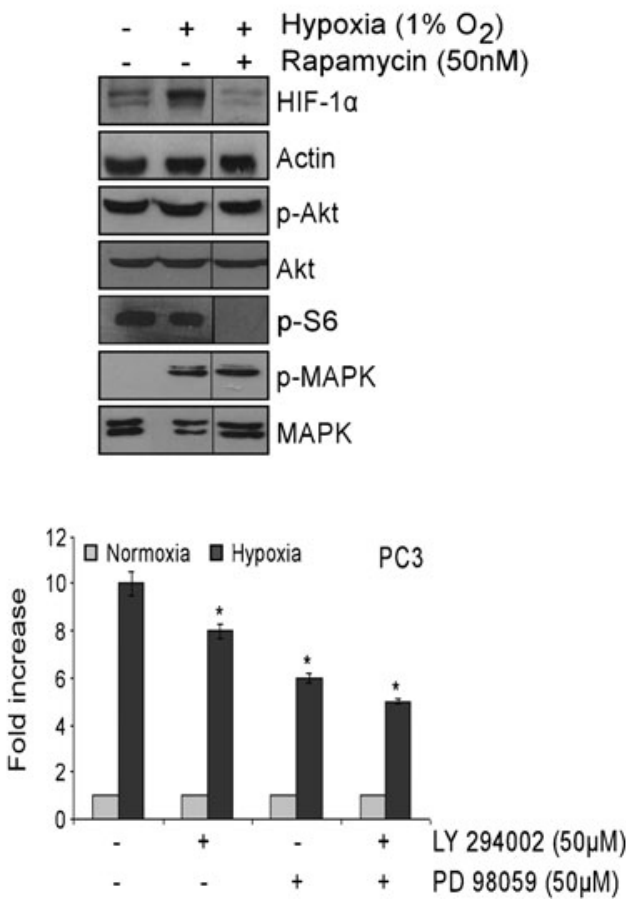
a

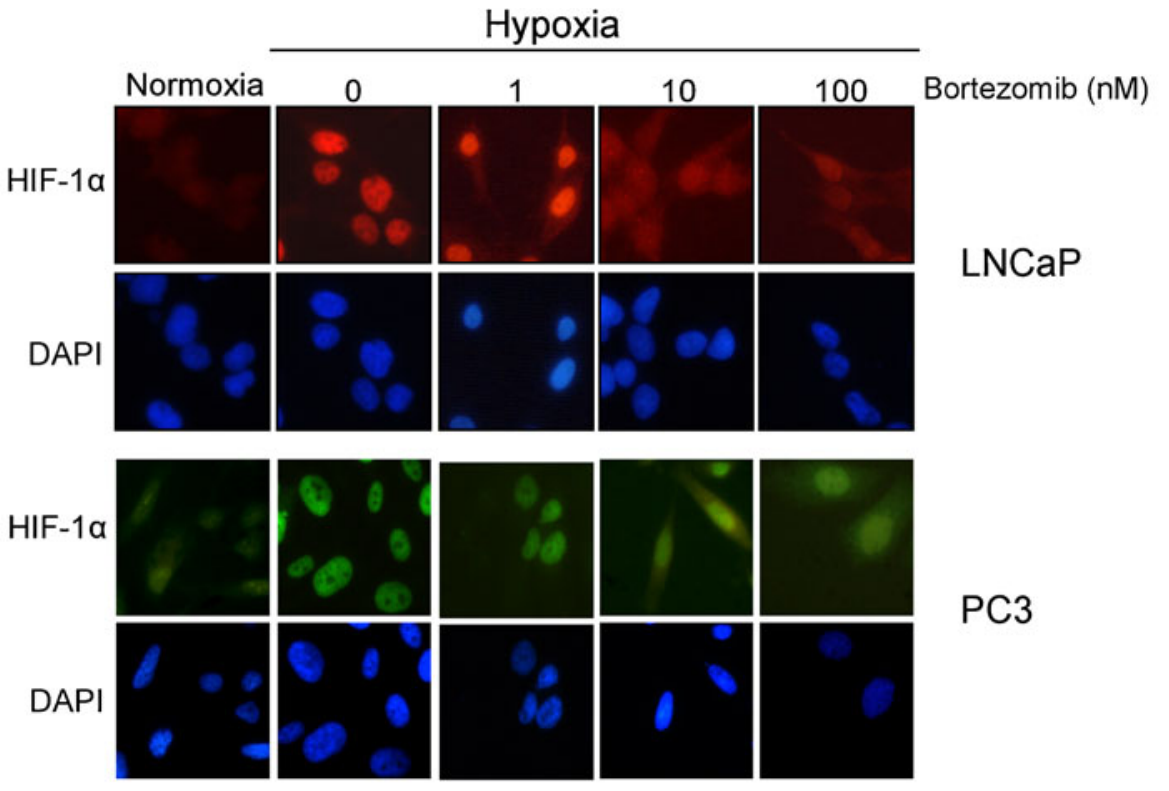

b

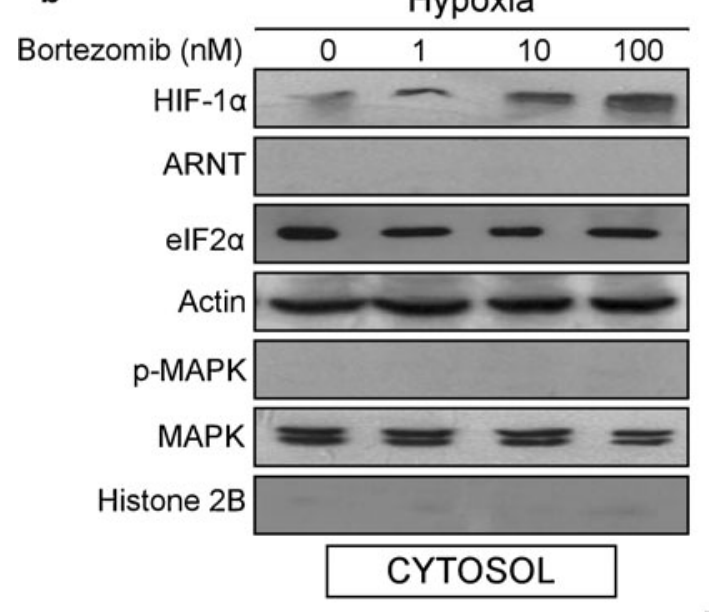

Hypoxia

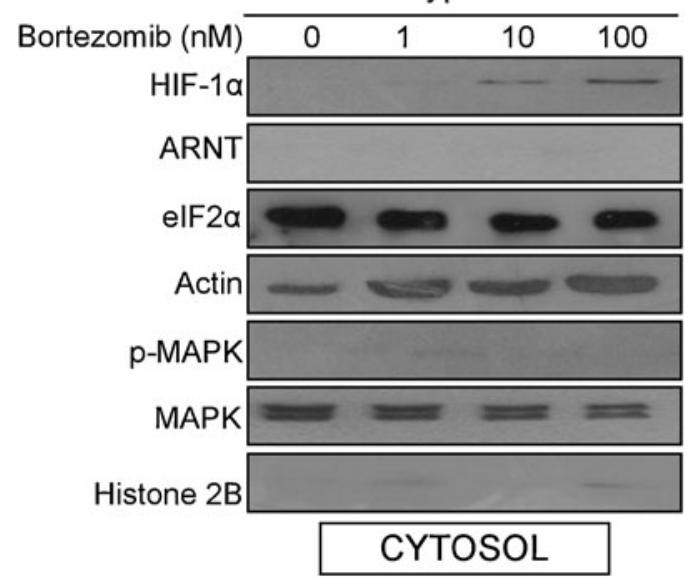

LNCaP

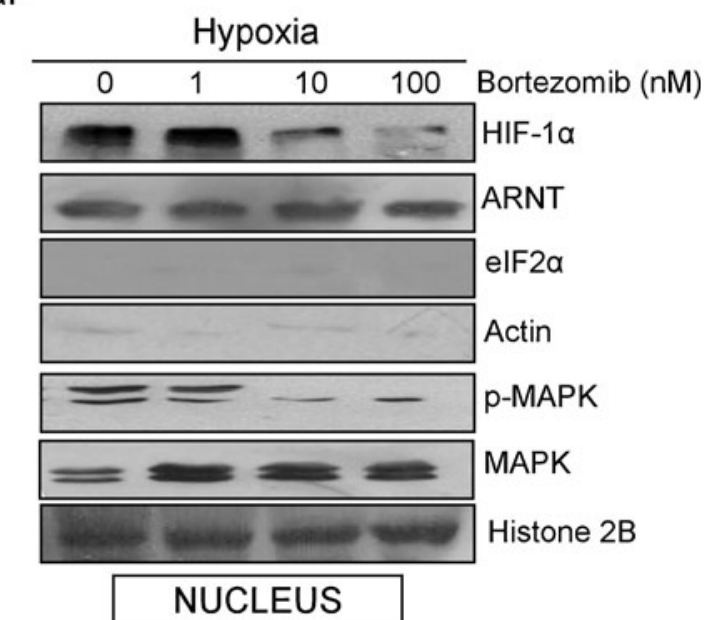

PC3

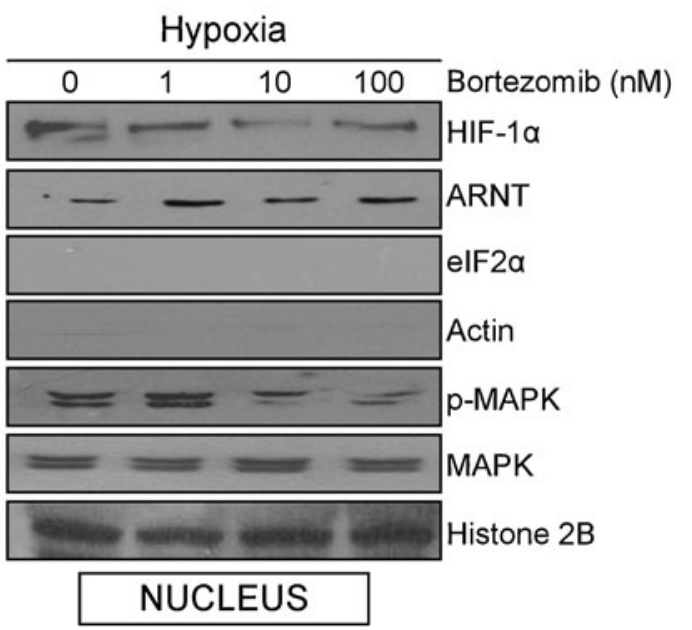

